\title{
Structural basis of SETD3 as an actin histidine methyltransferase
}

\author{
Shaobo Dai ${ }^{1}$, John R. Horton ${ }^{1}$, Xing Zhang ${ }^{1}$, Alex W. Wilkinson ${ }^{2}$, Or Gozani ${ }^{2}$, Xiaodong Cheng ${ }^{1}$ \\ 1. Department of Molecular and Cellular Oncology, The University of Texas MD Anderson Cancer \\ Center, Houston, TX 77030, USA \\ 2. Department of Biology, Stanford University, Stanford, CA, USA.
}

\section{Email Contact: sdai@mdanderson.org}

Abstract: As one of the most ubiquitous proteins, actin is known for methylation at histidine 73 for over fifty years ${ }^{1}$. However, the origin of this modification has remained elusive and the enzyme responsible for this methylation has not been identified. In collaboration with Dr. Alex W. Wilkinson, Dr. Or Gozani and colleagues of Standard University, we have characterized SETD3 as the specific actin histidine 73 methyltransferase that methylates the histidine's imidazole ring nitrogen N\&2 atom. We have determined the crystal structure of SETD3 in complex with $S$-adenosyl-L-homocysteine (SAH) and actin peptide (residues 66-80) at a resolution of $1.78 \AA^{2}$. The bound peptide adapts an extended conformation with the histidine imidazole inserting into the active site where the methyl donor is located. SETD3 shares the most similarity to SETD6 which is a lysine methyltransferase ${ }^{3}$ and structural alignment of both enzymes have revealed significant features which may contribute to the specific activity of SETD3 towards histidine. This work has expanded the well-characterized SET-domain catalyzed lysine methylation reaction to histidine methylation and highlights the structure of the first identified mammalian protein histidine methyltransferase complexed with peptide substrate and cofactor.

\section{References:}

1. Terman, J.R. and Kashina, A., 2013. Post-translational modification and regulation of actin. Current opinion in cell biology, 25(1), pp.30-38.

2. Wilkinson, A.W., Diep, J., Dai, S., Liu, S., Ooi, Y.S., Song, D., Li, T.M., Horton, J.R., Zhang, X., Liu, C. and Trivedi, D.V., Ruppel K.M., Vilches-Moure J.G., Casey K.M., Mak J., Cowan T., Elias J.E., Nagamine C.M., Spudich J.A., Cheng X., Carette J. E., Gozani, O., 2019. SETD3 is an actin histidine methyltransferase that prevents primary dystocia. Nature, 565(7739), p.372.

3. Chang, Y., Levy, D., Horton, J.R., Peng, J., Zhang, X., Gozani, O. and Cheng, X., 2011. Structural basis of SETD6mediated regulation of the NF-kB network via methyl-lysine signaling. Nucleic acids research, 39(15), pp.6380-6389. 